\title{
Higher Accurate Estimation of Axial and Bending Stiffnesses of Plates Clamped by Bolts*
}

\author{
Tomohiro NARUSE** and Yoji SHIBUTANI*** \\ **Hitachi, Ltd. Hitachi Research Laboratory, Mechanical Engineering Research Centre \\ 832-2, Horiguchi, Hitachinaka, Ibaraki, 312-0034, Japan \\ E-mail: tomohiro.naruse.az@hitachi.com \\ ${ }^{* * *}$ Department of Mechanical Engineering, Osaka University, \\ 2-1, Yamadaoka, Suita, Osaka, 565-0871, Japan
}

\begin{abstract}
Equivalent stiffness of clamped plates should be prescribed not only to evaluate the strength of bolted joints by the scheme of "joint diagram" but also to make structural analyses for practical structures with many bolted joints. We estimated the axial stiffness and bending stiffness of clamped plates by using Finite Element (FE) analyses while taking the contact condition on bearing surfaces and between the plates into account. The FE models were constructed for bolted joints tightened with M8, 10, 12 and 16 bolts and plate thicknesses of 3.2, 4.5, 6.0 and $9.0 \mathrm{~mm}$, and the axial and bending compliances were precisely evaluated. These compliances of clamped plates were compared with those from VDI 2230 (2003) code, in which the equivalent conical compressive stress field in the plate has been assumed. The code gives larger axial stiffness for $11 \%$ and larger bending stiffness for $22 \%$, and it cannot apply to the clamped plates with different thickness. Thus the code shall give lower bolt stress (unsafe estimation). We modified the vertical angle tangent, $\tan \varphi$, of the equivalent conical by adding a term of the logarithm of thickness ratio $t_{1} / t_{2}$ and by fitting to the analysis results. The modified $\tan \varphi$ can estimate the axial compliance with the error from $-1.5 \%$ to $6.8 \%$ and the bending compliance with the error from $-6.5 \%$ to $10 \%$. Furthermore, the modified $\tan \varphi$ can take the thickness difference into consideration.
\end{abstract}

Key words: Bolted Joint, Finite Element Method, Stress Analysis

\section{Introduction}

In a bolted joint, the bolt and the clamped plates are deformed as one body under working loads, such as external forces and external moments. Under these deformation conditions, external forces and moments are divided into additional load on the bolt and that of clamped plates in proportion to the stiffness ratio of bolt and clamped plates. For example, the bolted joint subjected to offset axial force is estimated by the superposition of axial force on the bolt axis and bending moment ${ }^{(4)}$. Thus, the stiffnesses of clamped plates under external force and moment are crucial in strength design guidelines ${ }^{(1)-(4)}$ of bolted joints. To evaluate the deformation or the vibration characteristic of industrial product structures jointed with a large number of bolts by using finite element (FE) analyses, what is needed is a simplified bolted-joints analysis model, in which equivalent stiffnesses of bolted joint are used, with a small number of elements ${ }^{(5) \sim(10)}$. For example, the bending stiffness of

*Received 17 Jan., 2012 (No. T2-09-7047) Japanese Original : Trans. Jpn. Soc. Mech. Eng., Vol.76, No.770, A (2010), pp.1234-1240 (Received 4 Dec., 2009) [DOl: 10.1299/jmmp.6.397]

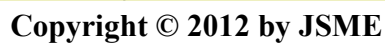


a bolted joint affects the natural frequency of the structure jointed with bolts ${ }^{(9)}$. Therefore, the FE model and the strength evaluation of bolted joints require that the stiffnesses of clamped plates under axial load, bending moment and torsional moment are precisely estimated.

We previously calculated the stiffnesses of clamped plates under axial load and bending moment by using FE models taking contact condition between clamped plates into consideration $^{(11)}$. The stiffnesses calculated by FE analyses agreed well with the German engineering society code, VDI $2230(2003)^{(4)}$, in which equivalent conical compressive stress fields in the plates had been assumed. However, this code gave the maximum difference of $11 \%$ large axial stiffness. In addition, the code had much quantitative error when applied in the case of clamped plates with different thicknesses. When the stiffness of clamped plates is estimated to be comparatively small, internal force borne to the bolt is predicted to be slightly smaller than the actual internal force. Accordingly the code gives a slightly unsafe estimation. Thus, the stiffness calculation should be improved.

In this paper, the equivalent stiffness model of clamped plates in VDI 2230 (2003) was improved to make it able to precisely estimate the stiffness in the case of clamped plates with different thickness.

\section{Stiffnesses of clamped plate}

Figure 1 schematically shows a bolted joint. When two plates are joined with sufficient clamping force and the contact conditions between the two plates are maintained under external force, the subjected external load is transmitted from one plate to the other through the clamp solid shown in the figure. The stiffness of the clamped plates, therefore, is expressed as the stiffness of the clamp solid.

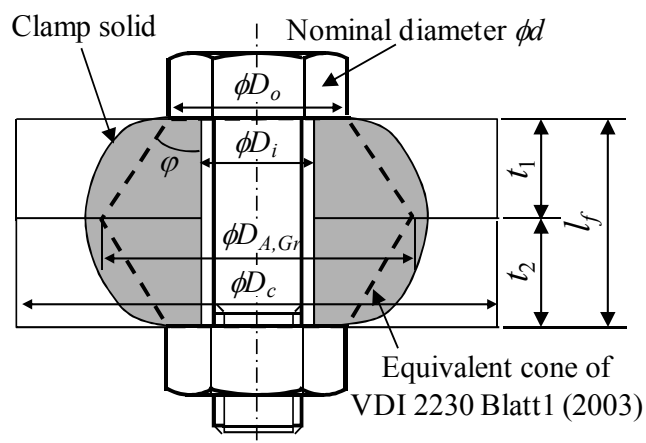

Fig. 1 Equivalent conical compressive stress field of plates clamped by bolted joint

According to VDI 2230 (2003) ${ }^{(4)}$, the clamp solid is assumed to be an equivalent cone as shown with dashed lines in Fig. 1. For a through bolted joint, the helix angle of the equivalent cone, $\varphi$, is obtained as follows:

$$
\tan \varphi=0.362+0.032 \ln \left(\frac{l_{f}}{2 D_{o}}\right)+0.153 \ln \left(\frac{D_{c}}{D_{o}}\right) .
$$

In this equation, $D_{o}$ is the outer diameter of the head-bearing surface, $l_{f}$ is the clamping length calculated by adding the thicknesses of two clamped plates, and $D_{c}$ is the outer diameter of the clamped plates. The maximum diameter of equivalent cone $D_{A, G r}$ is calculated as follows:

$$
D_{A, G r}=D_{o}+w l_{f} \tan \varphi \text {. }
$$

in which $w$ is called the joint coefficient and $w=1$ for a through bolted joint. When the clamped plates are large enough such as $D_{c}>D_{A, G r}$, the axial compliance of the clamped 
plates, $\delta_{c}$, is obtained as follows:

$$
\delta_{c}=\frac{2 \ln \left[\frac{\left(D_{o}+D_{i}\right)\left(D_{o}+w l_{f} \tan \varphi-D_{i}\right)}{\left(D_{o}-D_{i}\right)\left(D_{o}+w l_{f} \tan \varphi+D_{i}\right)}\right]}{w E_{c} \pi D_{i} \tan \varphi} .
$$

in which $D_{i}$ is the hole diameter, and $E_{c}$ is Young's modulus of the clamped plates.

The bending stiffness is calculated from the second moment of the equivalent cone area as follows:

$$
I_{\text {Bers }}=\frac{3 \pi}{64} \frac{\left(D_{c}-D_{o}\right) D_{o}^{3} D_{c}^{3}}{D_{c}^{3}-D_{o}^{3}}-\frac{\pi}{64} D_{i}^{4} .
$$

When the clamped plates are large enough such as $D_{c}>D_{A, G r}, D_{A, G r}$ is substituted for $D_{c}$ in Eq. (4). Thus the bending compliance of the clamped plates, $\beta_{c}$, is defined in the following equation:

$$
\beta_{c}=\frac{l_{f}}{E_{c} I_{\text {Bers }}} .
$$

\section{Analysis Method}

\subsection{Analysis model}

The analysis model of the previous study ${ }^{(11)}$ was used in this study as well. We estimated the stiffness of the clamped plates under clamping force, axial external force, and bending moment by analysing something with a 3D FE model that took the contact conditions between the clamped plates into consideration. Figure 2 shows the analytical model and its material properties. Figure 3 shows the 3D FE model. The analysis model consisted of two steel plates clamped by a steel bolt. The clamped plates and bolt had the elastic material properties of steel. The upper and lower bearing surfaces and the surface between the plates were covered with contact elements, and the friction coefficient of these contact surfaces was $\mu=0.15$. The plate thicknesses $t_{1}$ and $t_{2}$ were $3.2,4.5,6.0$ or $9.0 \mathrm{~mm}$ (a thickness of $3.2 \mathrm{~mm}$ being expressed as $t 3.2$ ), and $t_{1}$ and $t_{2}$ were varied with all combinations. The bolt section on the surface between the clamped plates was fixed to the axial direction ( $z$ direction). Moreover the cross point of the bolt axis and the surface between the plates was constrained to the $x$ direction. We used a half model because of the symmetry of the bending moment, and the symmetric sections of the bolt and the clamped plates were fixed to the $y$ direction. The diameter of the clamped plates was equal to $5 D_{o}$ so as not to affect the contact pressure distribution between the clamped plates. Analyses were carried out with ANSYS ${ }^{\circ}$ ver. 11 .

Table 1 shows some of the dimensions in Fig. 2, the clamping force (preload) $F_{f}$, the thread stress under the preload, $\sigma_{z S}$, and the average pressure on the bearing surface under the preload, $p_{w}$. Nominal bolt diameter was varied from M8 to M16, and the material strength of the bolts was 4.8 of ISO grade. The bolts were assumed to be full thread bolts, and the bolt model was assumed to be a cylinder with the minor diameter of the bolt thread. The hole diameter was defined as Class 2 value in JIS B1001. The outer diameter of the bearing surface was defined as being equal to that of a hexagon head with a washer stipulated in JIS B1180. The nut and bolt heights were equal to that of the hexagon head bolt specified in JIS B1180, though the nut is higher than the bolt head in the actual bolt. The preloads were given so as to be $70 \%$ of the yield pretension defined in JIS B1083. Pretension elements of ANSYS ${ }^{\circledR}$ were applied to the bolt section to give the preload. The thread stresses were $220 \mathrm{MPa}$. On the other hand the average pressure on the bearing surface varied from $166 \mathrm{MPa}$ to $238 \mathrm{MPa}$ in accordance with the nominal diameter of the bolt. 


\subsection{Load condition}

The axial force and bending moment similar to those in the previous study ${ }^{(11)}$ were supplied to the analysis models as shown in Figs. 2 and 3, and the axial and bending stiffness values of the clamped plates were estimated. We aimed to estimate the ideal stiffness of the clamped plates such that the entire external load would contributes to deformation on the bearing surface of the clamped plates. The load, therefore, was applied within the bearing surface.

Figure 4 shows the load conditions of the axial force. Negative pressure, which corresponds to the axial force $W_{a}$, was loaded on the bearing surfaces of the upper and lower clamped plates.

Figure 5 shows the load condition of the bending moment. When a bending moment that is a non-axisymmetric load was applied to an axisymetric model (such as a cylinder) with axisymmetric elements, it is expanded into the first order Fourier series. Consequently, the result obtained is the first order Fourier integral of the axisymmetric results ${ }^{(13)}$. In this study, 3D solid models were used because nonlinear analyses including contact conditions were carried out. The bending moment was modelled as pressure $p$ of a first order Fourier function, i.e., a cosine function of the angle $\theta$, that is $p=100 \cos \theta[\mathrm{MPa}]$ as shown in Fig. 5 . The minimum pressure of $-100 \mathrm{MPa}$ was smaller than the bearing pressure under the preload $p_{w}$ shown in Table 1. This may not open the contact surface between the clamped plates on the tension side.

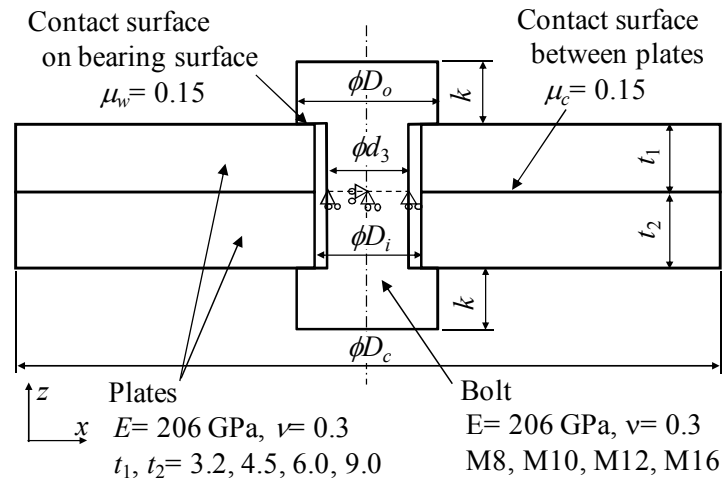

Fig. 2 Dimensions and material properties of analysis model [mm]

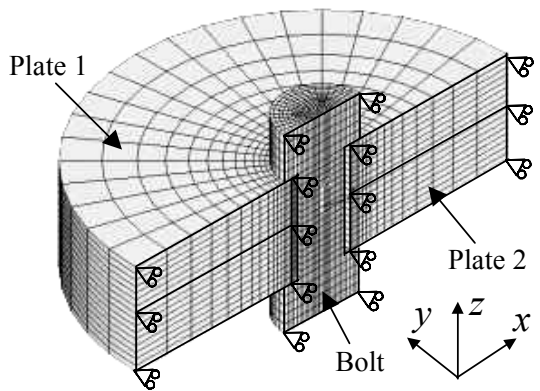

Fig. 3 3D finite element model of bolted joint

Table 1 Dimensions of analysis model and preload

\begin{tabular}{|ll|r|r|r|r|}
\hline & & M8 & \multicolumn{1}{|c|}{ M10 } & M12 & \multicolumn{1}{c|}{ M16 } \\
\hline$d_{3}$ & {$[\mathrm{~mm}]$} & 6.647 & 8.376 & 10.106 & 13.835 \\
\hline$D_{i}$ & {$[\mathrm{~mm}]$} & 9 & 11 & 13.5 & 17.5 \\
\hline$D_{o}$ & {$[\mathrm{~mm}]$} & 11.63 & 14.63 & 16.63 & 22 \\
\hline$k$ & {$[\mathrm{~mm}]$} & 5.3 & 6.4 & 7.5 & 10 \\
\hline$F_{f}$ & {$[\mathrm{~N}]$} & 7,670 & 12,130 & 17,610 & 32,620 \\
\hline$\sigma_{z S}$ & {$[\mathrm{MPa}]$} & 221 & 220 & 220 & 217 \\
\hline$p_{w}$ & {$[\mathrm{MPa}]$} & 180 & 166 & 238 & 234 \\
\hline
\end{tabular}




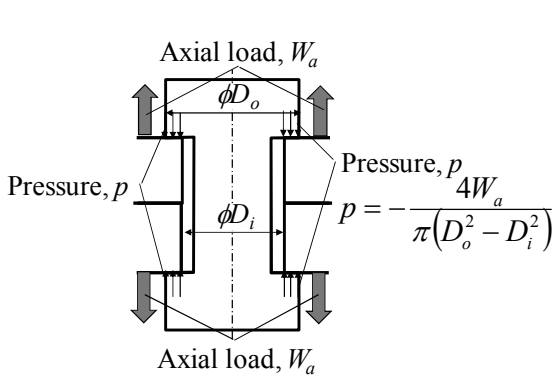

Fig. 4 Axial load condition of analysis model

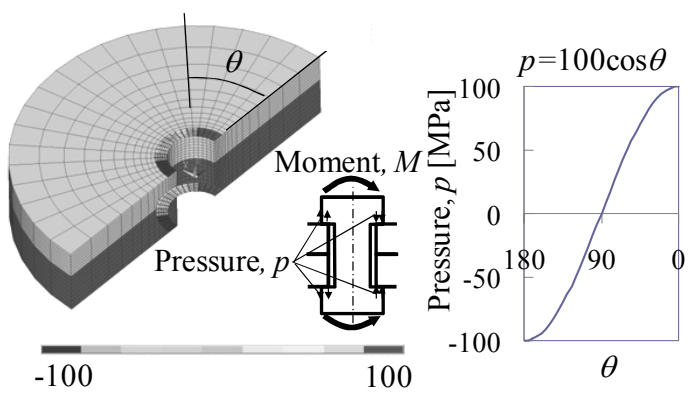

Fig. 5 Bending moment condition of analysis model

\section{Analysis Result}

The analysis model in Figs. 2 and 3 was subjected to preload of bolt first and then the axial force as shown in Fig. 4 second. The compression force of the clamped plates was calculated by integrating the contact pressure between the clamped plates in the contact area. The deformation of the bearing surface was estimated as the average of the deformation at the outer bearing diameter and that at the hole diameter, though the bearing surface deformed like a cone-shape. The compression deformation of the clamped plates was set to the deformation difference between the upper and lower bearing surface. The axial compliance $\delta_{c}$ under the axial load could be calculated from the slope of the relationship between the compression force and the compression deformation. The axial compliance did not depend on the diameter at which the deformation of the clamped plates was estimated ${ }^{(11)}$. For example, the axial compliance $\delta_{c}$ of analysis results and that calculated by Eq. (1) of VDI 2230 (2003) were compared for an M10 bolt by varying the clamping length $t_{1}+t_{2}$ as shown in Fig. 6.

Next, the bending compliances of the clamped plates under the bending moment were evaluated with the analysis models in Figs. 2 and 3 those were subjected to the load condition in Fig. 5. The bending moment of the clamped plates was obtained as a surface integral of the product of the contact pressure between the clamped plates and the $x$ coordinate in the contact area. The bending compliance was derived from the slope of the relationship between the bending moment of the clamped plates and the incline difference between the upper and lower bearing surface. For example the bending compliance $\beta_{c}$ of analysis results and those calculated by Eqs. (1), (4) and (5) of VDI 2230 (2003) were compared for an M10 bolt by varying the clamping length $t_{1}+t_{2}$ as shown in Fig. 7.

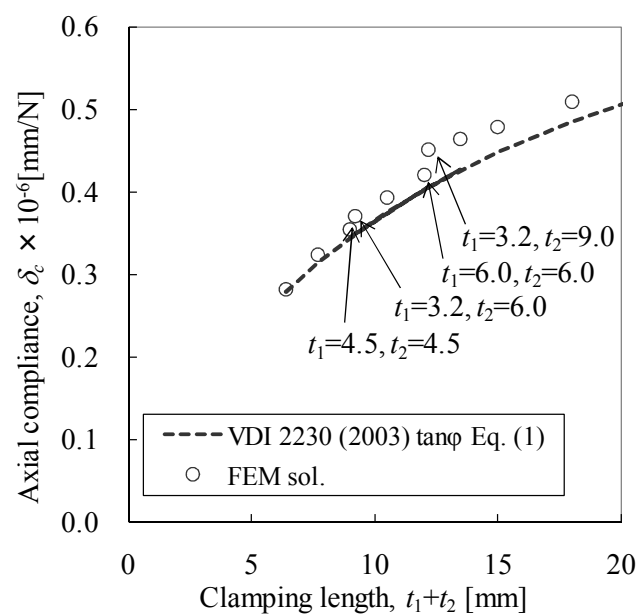

Fig. 6 Axial compliance of clamped plates with M10

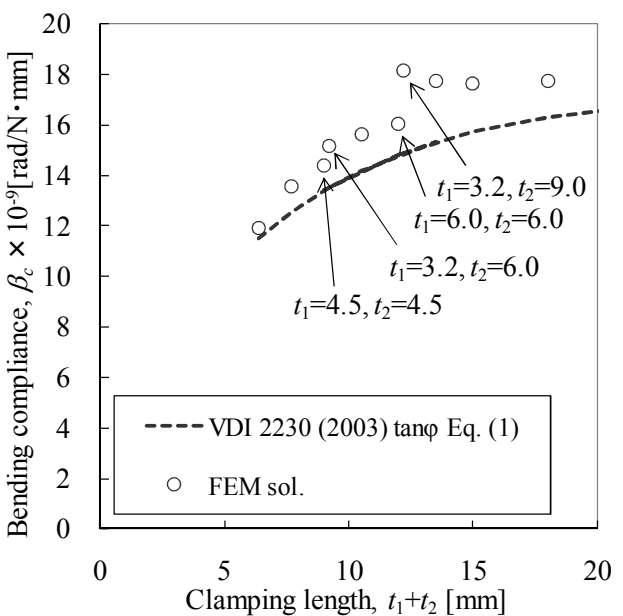

Fig. 7 Bending compliance of clamped plates with M10 
The axial and bending compliances of analysis results agreed well with the values of VDI 2230 (2003). However, the analysis results were 11\% larger than the value of VDI 2230 (2003) for the axial compliance and 22\% larger for the bending compliance in some cases. VDI 2230 (2003) may give higher stiffness because it was evaluated from an analysis model that fixed the surface between the clamped plates. If the stiffness of the clamped plates were estimated to be higher than the actual stiffness, i.e. lower compliance than the actual compliance, the bolt stress would be estimated to be lower than the actual stress and this would provide an unsafe result. VDI 2230 (2003), therefore, may turn out to provide an unsafe evaluation.

The compliances of the analysis results were two values at the clamping lengths $t_{1}+t_{2}$ close to $9 \mathrm{~mm}$ and $12 \mathrm{~mm}$. These were obtained with $t_{1}=t_{2}=4.5$ and $t_{1}=3.2, t_{2}=6.0$ for $t_{1}+t_{2}=9$, and with $t_{1}=t_{2}=6$ and $t_{1}=3.2, t_{2}=9.0$ for $t_{1}+t_{2}=12$, respectively. It shows that the compliance with plates that have the same thicknesses was different from that with plates that have different thicknesses. Plates with different thicknesses gave higher compliance values than those with the same thicknesses. When the clamped plates had very different thickness, their stiffnesses were lower than those given in VDI 2230 (2003), in which the thicknesses are assumed to be the same. This is because the thin plates exhibit large deformation and provide low stiffness values.

\section{Improvement of the stiffness of the clamped plates}

In the previous chapter, VDI 2230 (2003) gave larger stiffnesses of the clamped plates than the analysis results. Moreover, the larger the difference in the clamped plates thicknesses, the greater the stiffness difference between the analysis result and VDI 2230 (2003). The helix angle of the equivalent cone, $\tan \varphi$, is based on the analysis results of Lori et al. ${ }^{(9)}$, who analysed the model shown in Fig. 8. Lori et al. determined the parameters of Eq. (1) for $\tan \varphi$ by using multi regression analysis with the FE results, in which the dimensions of the bolt and clamped plate varied widely such as $l_{f} / D_{o}=0.59 \sim 4.82$. In this analysis model, both clamped plates had the same thickness and the analysis model was assumed to be vertically symmetric; the contact surface between two clamped plates was fixed. Thus, the contact surface between the clamped plates could not separate, and the stiffness of the clamped plates was thought to have been estimated higher than the actual stiffness. Furthermore, this model could not take into consideration the thickness difference between the clamped plates.

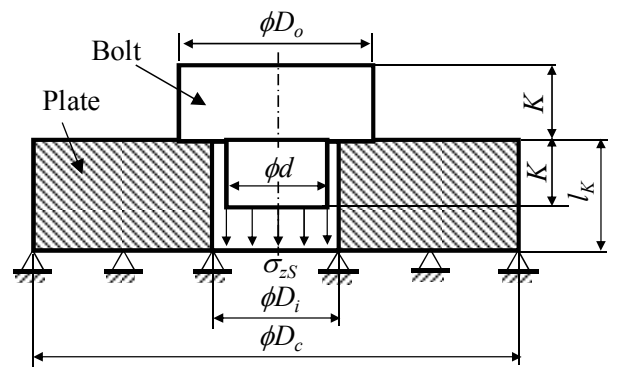

Fig. 8 Lori's model of finite element analysis ${ }^{(9)}$

In this study, we estimated the axial compliance with the model that took the contact condition between the clamped plates into account as shown in Figs 2 and 3. Moreover, this model could evaluate the model of the clamped plates with different thicknesses. On the other hand, the model was restricted to thinner clamped plates, such as $l_{f} / D_{o}=0.11 \sim 1.29$, than Lori's model. We estimated the value of $\tan \varphi$ with the axial compliance of the analysis result as shown in Fig. 6 by using an iterative method, namely quasi Newton's method. 
The relationship between the value of $\tan \varphi$ calculated with the axial compliance of the analysis result and the thickness ratio $t_{1} / t_{2}$ (in which $t_{1}<t_{2}$ ) is shown in Fig. 9. The value of $\tan \varphi$ was found to increase as the thickness ratio $t_{1} / t_{2}$ increased. To compare clearly this analysis result of $\tan \varphi$ and the value of $\tan \varphi$ calculated by Eq. (1), the difference between these two $\tan \varphi$ values was evaluated. The relationship between the difference in two $\tan \varphi$ values and the thickness ratio $t_{1} / t_{2}$ is shown in Fig. 10. The analysis results of $\tan \varphi \operatorname{are}$ roughly divided into two types, that of M8 and M16 and that of M10 and M12. For both types, the analysis result of $\tan \varphi$ increases as the thickness ratio $t_{1} / t_{2}$ increases and the difference in the analysis result and VDI 2230 (2003) shrinks as the thickness ratio $t_{1} / t_{2}$ moves closer to one.

In Fig. 10, the approximated equations with logarithmic function of $t_{1} / t_{2}$ are written for the four analysis results from M8 to M16. All the four analysis results are clearly represented with logarithmic functions of $t_{1} / t_{2}$. The coefficients of $\ln \left(t_{1} / t_{2}\right)$ with the approximated equations, which are within the region from 0.0625 to 0.0764 , do not differ much, and the average value is 0.0717 . The constant terms of the approximated equations represent the differences from VDI 2230 (2003) for $t_{1}=t_{2}$. The larger the absolute value of the negative constant term, the lower the stiffness. The constant terms of M10 and M12 are negative such as -0.039 , which is the stiffness of those lower than VDI 2230 (2003). On the other hand, the constant terms of M8 and M16 are positive.

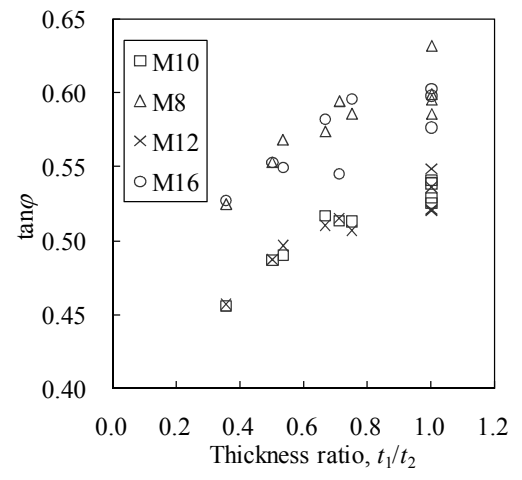

Fig. 9 Relationship between thickness ratio and analysis result of $\tan \varphi$

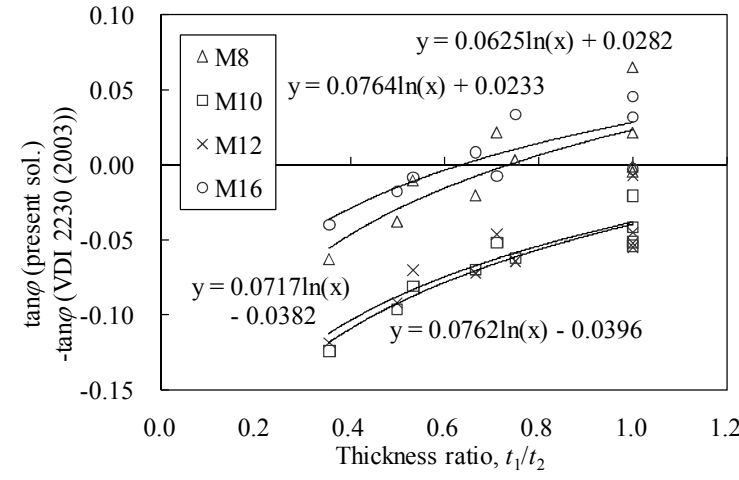

Fig. 10 Relationship between thickness ratio and difference of $\tan \varphi$

From the above consideration for the analysis result of $\tan \varphi$, we improved an equation of $\tan \varphi$ from Eq. (1) to Eq. (6).

$$
\tan \varphi=0.323+0.032 \ln \left(\frac{l_{f}}{2 D_{o}}\right)+0.153 \ln \left(\frac{D_{c}}{D_{o}}\right)+0.0717 \ln \left(\frac{t_{1}}{t_{2}}\right) \quad\left(\text { when } t_{1} \leq t_{2}\right)
$$

In Eq. (6), the logarithmic functions, $0.0717 \ln \left(t_{1} / t_{2}\right)$, in which 0.0717 is the average value of the coefficients of $\ln \left(t_{1} / t_{2}\right)$ in the approximated equations of Fig. 10, is added to Eq. (1). When $t_{1}=t_{2}, \ln \left(t_{1} / t_{2}\right)$ becomes zero, and the form of Eq. (6) is coincident with that of Eq. (1) of VDI 2230 (2003).

Moreover, we modified the constant term with -0.039 , because VDI 2230 (2003) had given higher stiffness for M10 and M12 than the actual stiffness and the code had estimated bolt force slight unsafely. Thus, the constant term of Eq. (6) becomes 0.323 by subtracting 0.039 from 0.362 of the constant term of Eq. (1).

The axial compliances calculated with the modified $\tan \varphi$ of Eq. (6) were compared with those of the FE solution and those calculated with Eq. (1) of VDI 2230 (2003) as shown from Fig. 11 to Fig. 14. The modified $\tan \varphi$ of Eq. (6) agreed well with the FE solution for M10 and M12, though VDI 2230 (2003) gave higher stiffness. The modified $\tan \varphi$ of Eq. (6) gave slightly higher compliance, i.e. slightly lower stiffness, than the FE solution for M8 and M16. The modified $\tan \varphi$ of Eq. (6) could well represent the effect of plate thickness 
difference on the compliance caused by the effect of the logarithmic functions of $t_{1} / t_{2}$, though VDI 2230 (2003) could not. Consequently, the modified $\tan \varphi$ of Eq. (6) could precisely estimate the axial compliance within the errors from $-1.5 \%$ to $6.8 \%$ for the FE solutions, even though VDI 2230 (2003) gave higher error for $-11 \%$ low compliance.

The bending compliances calculated with the modified $\tan \varphi$ of Eq. (6) by using Eqs. (4) and (5) were compared with those of the FE solution and those calculated with Eq. (1) of VDI 2230 (2003) as shown from Fig. 15 to Fig. 18. The modified $\tan \varphi$ of Eq. (6) agreed with the FE solutions better than VDI 2230 (2003), even though the modified $\tan \varphi$ of Eq. (6) gave slightly higher compliance for M8 and M16, and slightly lower compliance for M10 and M12. The modified $\tan \varphi$ of Eq. (6) could accurately represent the effect of plate thickness difference on the compliance, though VDI 2230 (2003) could not. Consequently, the modified $\tan \varphi$ of Eq. (6) could precisely estimate the bending compliance within the errors from $-6.5 \%$ to $10 \%$ for the FE solutions, even though VDI 2230 (2003) gave higher error for $-22 \%$ low compliance.

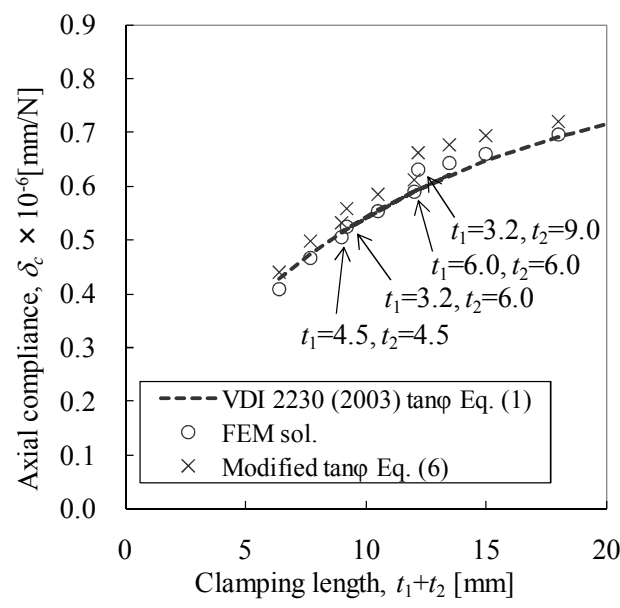

Fig. 11 Comparison of axial compliance of clamped plates with M8

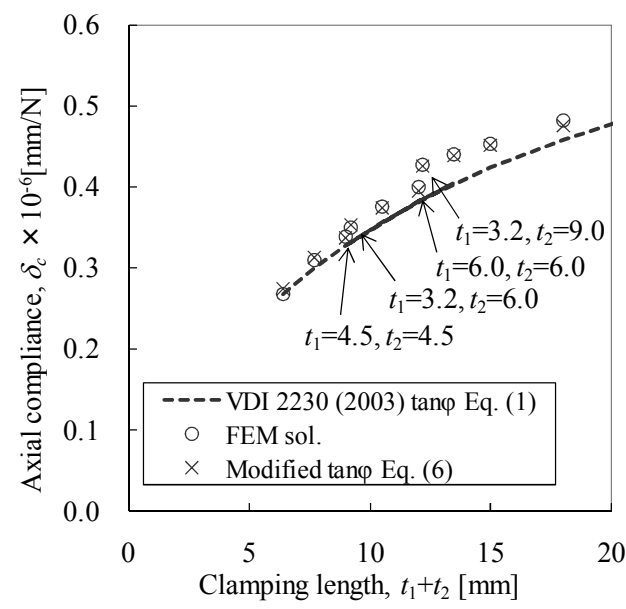

Fig. 13 Comparison of axial compliance of clamped plates with M12

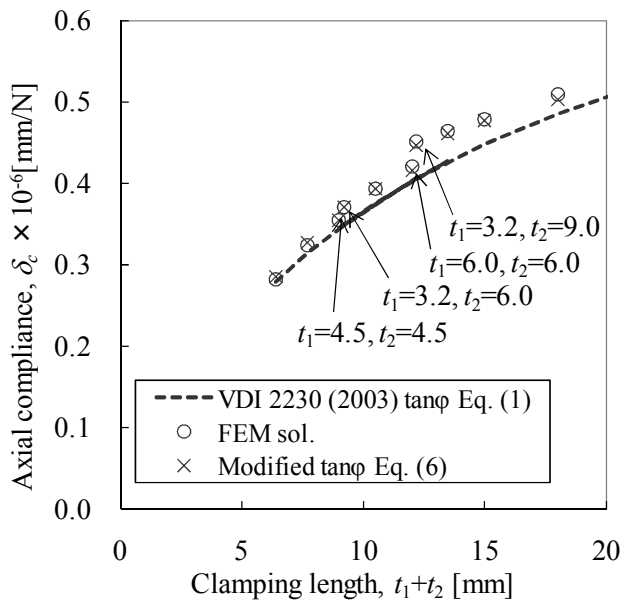

Fig. 12 Comparison of axial compliance of clamped plates with M10

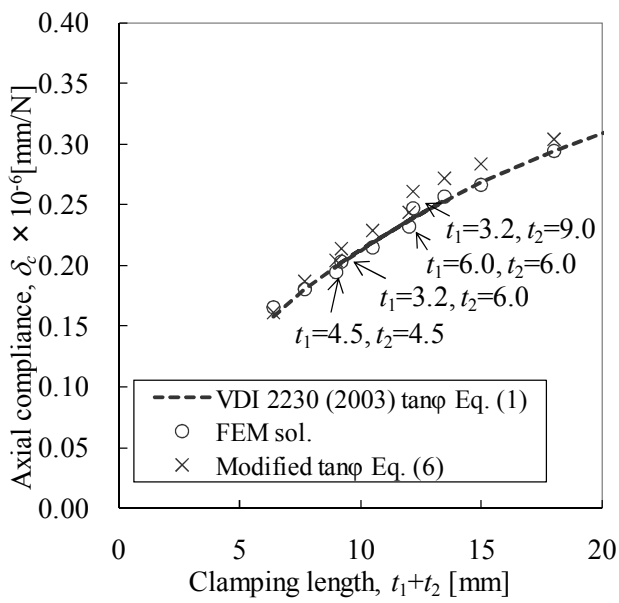

Fig. 14 Comparison of axial compliance of clamped plates with M16 


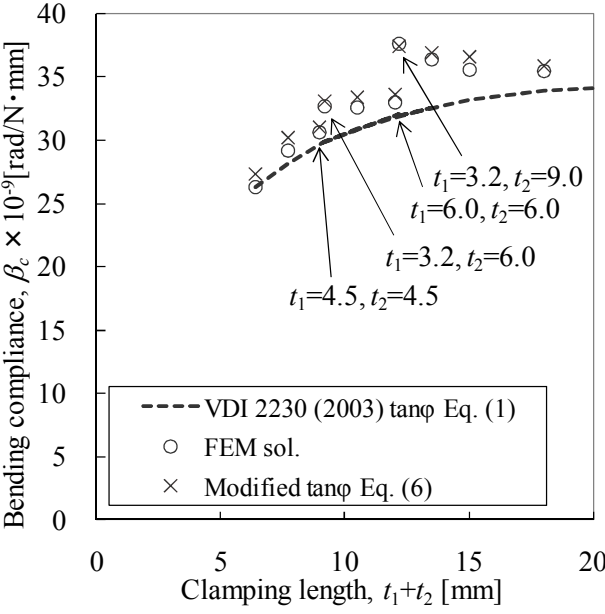

Fig. 15 Comparison of bending compliance of clamped plates with M8

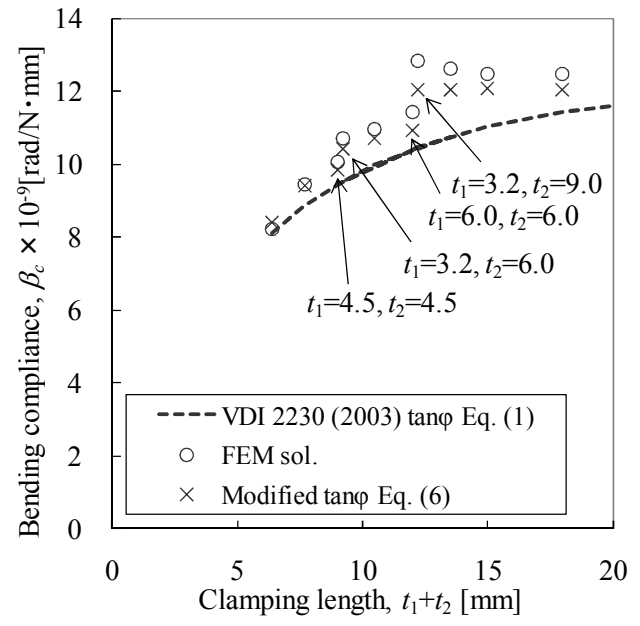

Fig. 17 Comparison of bending compliance of clamped plates with M12

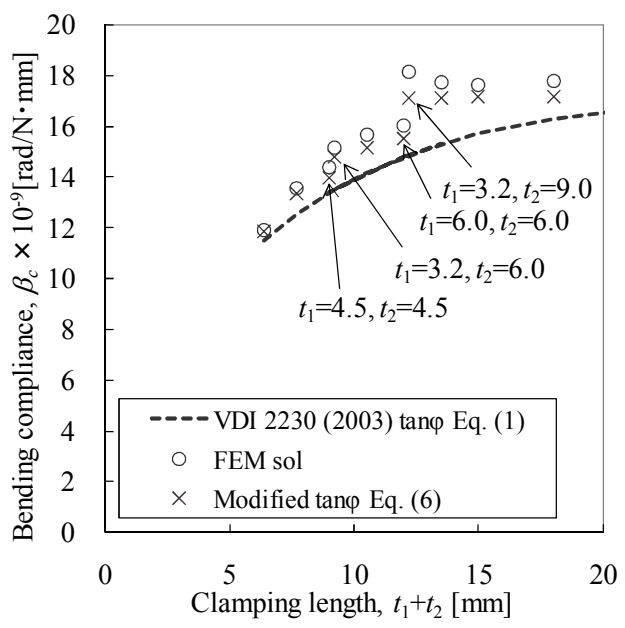

Fig. 16 Comparison of bending compliance of clamped plates with M10

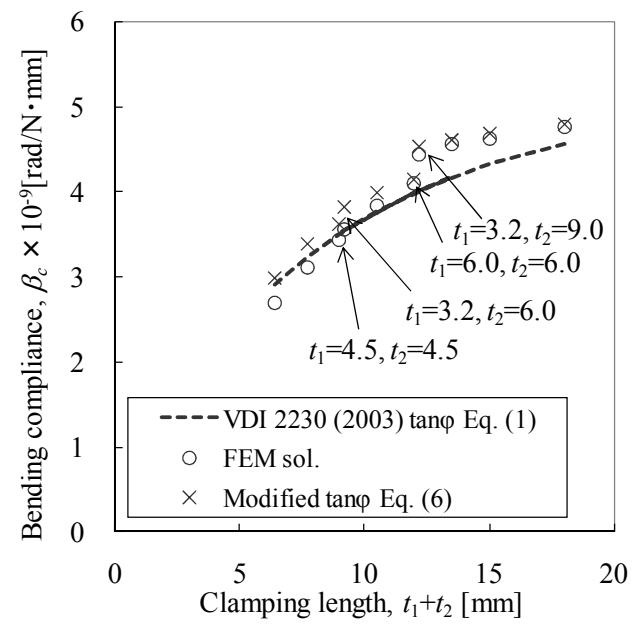

Fig. 18 Comparison of bending compliance of clamped plates with M16

\section{Conclusion}

We evaluated the axial and bending compliances of clamped plates by using finite element (FE) models of bolted joints taking the contact conditions into consideration. Moreover, we improved the equivalent stiffness of the clamped plates in present VDI 2230 (2003). The following results were obtained in our work:

(1) VDI 2230 (2003) specifies 11\% large axial stiffness and 22\% large bending stiffness in some several case, so the code gives slightly unsafe estimation. In addition the code gives large errors when applied in the case of clamped plates with different thicknesses due to the symmetry model assumed in the FE model of VDI 2230 (2003).

(2) The helix angle of the equivalent cone, $\tan \varphi$, was calculated from our FE results. According to the relationship between the analysis result of $\tan \varphi$ and the thickness ratio of $t_{1} / t_{2}$, we improved an equation of $\tan \varphi$ by adding the logarithmic functions of $\ln \left(t_{1} / t_{2}\right)$ and by modifying the constant term.

(3) When the modified equation of $\tan \varphi$ was applied to the bolted joints with $l_{f} / D_{o}=0.11 \sim 1.29$, the modified $\tan \varphi$ could precisely estimated the axial compliance within the errors from $-1.5 \%$ to $6.8 \%$, and the bending compliance within the errors from $-6.5 \%$ to $10 \%$. 


\section{References}

(1) Yamamoto, A., Theory and Design of Bolt Joints (in Japanese), (1977), pp. 30-69, Yokendo.

(2) Yoshimoto, I., Points for Design of Bolt Joints (in Japanese), (1992), pp. 177-215, Japanese Standards Association.

(3) Editorial board of handbook of joint technique, Handbook of Joint Technique (in Japanese), (1994), pp. 615-640, Industrial service centre.

(4) Richtlinien VDI 2230, B1., Systematic calculation of high duty bolted joints -Joints with one cylindrical bolt-, (2003), VDI-Verlag [Translated into Japanese by Gasei, S. and Kawai, K., (2006), The Japan Research Institute for Screw Threads and Fasteners].

(5) Kim, J. G., Park, S. S., Choi, S. H. and Kim, B. K., Finite Element Modelling and Experimental Verification of the Structures with Bolted Joints, Transactions of the Korea Society of Mechanical Engineers, Vol. 20 No. 6 (1996), pp. 1854-1861.

(6) Hurrell, P. R., Good Practice in Modelling of Pressure Vessel Bolted Joints for Stress and Fatigue Analysis, ASME PVP, Vol. 405 (2000), pp. 123-134.

(7) Montgomery, J., Methods for Modeling Bolts in the Bolted Joint, ANSYS 2002 Conference, 2002 April.

(8) Rutman, A. and Kogan, J. B., Software takes the load off joint modeling, Machine Design, Vol. 70 No. 6 (1998), pp.79-82.

(9) Naruse, T., Kawasaki, T. and Hattori, T., Simple Modelling and Strength Evaluation Methods for Bolt Joints Using Shell Elements and Beam Elements (1st Report, Modelling Method), Journal of Computational Science and Technology, Vol. 3 No.1 (2009), pp. 22-33 [Transactions of the Japan Society of Mechanical Engineers, Series A, Vol. 73 No. 728 (2005), pp.522-528].

(10) Naruse, T., Kawasaki, T. and Hattori, T., Simple Modelling and Strength Evaluation Methods for Bolt Joints Using Shell Elements and Beam Elements (2nd Report, Strength Evaluation Method), Journal of Computational Science and Technology, Vol. 3 No.1 (2009), pp. 34-45 [Transactions of the Japan Society of Mechanical Engineers, Series A, Vol. 73 No. 728 (2005), pp. 529-536].

(11) Naruse, T. and Shibutani, Y., Equivalent Stiffness Evaluations of Clamped Plates in Bolted Joints under Loading, Journal of Solid Mechanics and Materials Engineering, Vol. 4 No. 12 (2010), pp. 1791-1805 [Transactions of the Japan Society of Mechanical Engineers, Series A, Vol. 75 No. 757 (2009), pp. 1230-1238].

(12) Lori, W., and Glaser, H., Berechnung der Plattennachgiebigkeit bei Schraubenverbindungen, Konstruktion, Vol. 42 (1990), pp.271-277 [Translated into Japanese by Gasei, S, Journal of Japan Research Institute for Screw Threads and Fasteners, Vol. 22 No.12 (1991), pp.393-401].

(13) ANSYS v.11.0 Documentation, 2.13 Axisymetric Elements with Nonaxisymmetric Loads, ANSYS 